\title{
Broadband and Picosecond Intraband Absorption in Lead-Based Colloidal Quantum Dots
}

Bram De Geyter, ${ }^{+,}$Arjan J. Houtepen, ${ }^{\ddagger}$ Sergio Carrillo, ${ }^{5}$ Pieter Geiregat, ${ }^{+, \mp}$ Yunan Gao, ${ }^{*}, "$ Sybren ten Cate, Juleon M. Schins, ${ }^{\mp}$ Dries Van Thourhout, ${ }^{+{ }^{\top 9}}$ Christophe Delerue, ${ }^{5}$ Laurens D. A. Siebbeles, ${ }^{\ddagger}$ and Zeger Hens $\boldsymbol{s}^{\mathrm{I}, \mathrm{I}, *}$

${ }^{\dagger}$ Photonics Research Group, INIEC Department, Ghent University-IMEC, Sint-Pietersnieuwstraat 41, 9000 Ghent, Belgium, ${ }^{\ddagger}$ Optoeletronic Materials Section, Department of Chemical Engineering, Delft University of Technology, Julianalaan 136, 2628 BL Delft, The Netherlands, ${ }^{5}$ Department ISEN, CNRS-UMR 8520, Institute for Electronics, Microelectronics, and Nanotechnology (IEMN), FR-59652 Villeneuve d'Ascq, France, "Inorganic and Physical Chemistry Departement, UGent, Krijgslaan 281 (S3), B-9000 Gent, Belgium, "Center for Nano- and Biophotonics (NB-Photonics), Ghent University, 9000 Ghent, Belgium, and "The Kavli Institute of Nanoscience, Delft University of Technology, Lorentzweg 1, 2628 C Delft, The Netherlands

1 he tunability and ease of processing of colloidal quantum dots (QDs) offer many opportunities for the development of novel opto-electronic devices, such as lasers, electro-optic modulators, and highspeed detectors. ${ }^{1,2}$ The design of efficient and competitive materials for the photonics industry based on QDs requires a precise understanding of the electronic states involved in light-matter interaction and the decay dynamics of photogenerated carriers. In the case of QDs with their first exciton absorption in the near-infrared $(800-2000 \mathrm{~nm})$, most studies have focused on interband transitions and intraband relaxation. ${ }^{3-6}$ On the other hand, a few literature studies indicate that additional electronic transitions are relevant in excited QDs. Intraband absorption between the 15 and 1P state has been studied extensively for $\mathrm{CdSe}_{1}{ }^{7-9} \mathrm{PbSe}_{1}{ }^{6,10}$ and $\mathrm{PbS}_{1}^{11}$ with good agreement between experiment and theory. In the case of CdSe QDs, several authors ${ }^{12-15}$ reported photoinduced absorption (PA) at wavelengths directly below the band gap, which they attributed to absorption from excited trap states at the QD surface. Recent work on CdSe QDs ${ }^{16}$ and CdSe/CdS QDs ${ }^{17}$ identified absorption from holes to be the main contribution to this signal. For PbS QDs coupled to nanostructured metal oxides, ${ }^{18,19}$ a similar broad PA feature below the band gap was observed, though its origin remained unclear.

In this paper, we report on strong, broadband photoinduced absorption (PA) in lead chalcogenide QDs, starting at photon energies directly below the band gap. Using

\section{ABSTRACT}
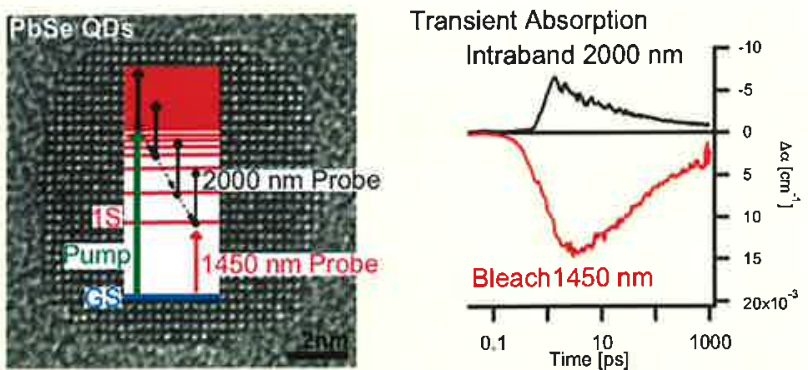

Using femtosecond transient absorption spectroscopy, we demonstrate that lead chalcogenide nanocrystals show considerable photoinduced absorption (PA) in a broad wavelength range just below the band gap. The time-dependent decay of the PA signal correlates with the recovery of the band gap absorption, indicating that the same carriers are involved. On this basis, we assign this PA signal to intraband absorption, that is, the excitation of photogenerated carriers from the bottom of the conduction band or the top of the valence band to higher energy levels in the conduction and valence band continuum. We confirm our experiments with tight-binding calculations. This broadband response in the commercially interesting near- to mid-infrared range is very relevant for ultra-high-speed all-optical signal processing. We benchmark the performance with bulk Si and Si nanocrystals.

KEYWORDS: intraband absorption - lead-based quantum dots - broadband silicon photonics $\cdot$ semiconductor $\cdot$ nanocrystals $\cdot \mathrm{PbSe} \cdot \mathrm{PbSe} / \mathrm{CdSe}$

femtosecond transient absorption (TA) spectroscopy, we find that the decay of the PA matches the recovery of the band *Address correspondence to zeger.hens@ugent.be. gap absorption. Therefore, we interpret the PA in terms of transitions of excited electrons and holes from the lowest conduction (LUMO) and the highest valence band state (HOMO) to the conduction and valence band continuum, respectively. Moreover, 


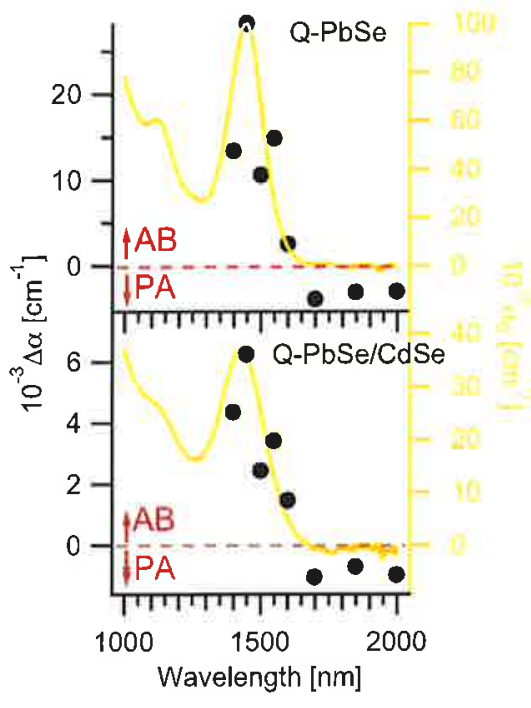

Figure 1. Steady state (orange line, right axis) and differential transient absorption spectrum (black dots, left axis) of $\mathrm{PbSe}$ QDs (top graph) and $\mathrm{PbSe} / \mathrm{CdSe}$ (bottom graph) $5 \mathrm{ps}$ after the pump pulse shows absorption bleach (AB) of the band gap absorption ( $\left.\Delta \alpha=\alpha_{0}-\alpha>0\right)$ and wavelength-independent photoinduced absorption (PA, $\Delta \alpha<0$ ) below the band gap.

we demonstrate that this broad intraband absorption is a characteristic of several $\mathrm{Pb}$ chalcogenide QDs, including $\mathrm{PbSe}$ and $\mathrm{PbS}$ core QDs and $\mathrm{PbSe} / \mathrm{CdSe}$ core/ shell QDs. Using tight-binding ${ }^{20}$ calculations on PbSe QDs, we show agreement between the observed PA and the calculated intraband absorption spectrum. The calculations shed light on the initial and final states involved in the photoinduced absorption process.

Because of intraband absorption, excited $\mathrm{Pb}$ chalcogenide QDs absorb light in a wavelength range where ground state QDs are transparent. Depending on the size of the QDs, this wavelength range can be tuned to cover wavelengths around 1300 and $1550 \mathrm{~nm}$, which are widely used in optical communication. Since intraband transitions have a strong absorption coefficient and are followed by picosecond intraband cooling, our findings show that $\mathrm{Pb}$ chalcogenide $\mathrm{QDs}$ are highly relevant materials for telecom applications. Free carrier absorption (FCA), the equivalent process in bulk semiconductors, is nowadays widely studied for optical modulation. ${ }^{21,22}$ However, momentum conservation forbids intraband transitions in this case, which makes a phonon needed for FCA. This results in a relatively low absorption coefficient and makes applications in ultrafast optical switching sensitive to the device temperature. Hence, by proving that intraband absorption is an allowed process in Pb chalcogenide QD5 over a broad wavelength range, our results are a particular example of how size quantization affects the properties of materials up to a level where they outperform bulk materials.

\section{RESULTS}

Figure 1 shows the change in absorption of $\mathrm{PbSe}$ core $(d=4.6 \mathrm{~nm})$ and $\mathrm{PbSe} / \mathrm{CdSe}$ core/shell QDs
( $4.5 \mathrm{~nm}$ core, $0.5 \mathrm{~nm}$ shell) following excitation with a $50 \mathrm{fs}$ pump pulse $\left(\lambda_{\text {pump }}=790 \mathrm{~nm}\right)$. This resulting excited state cools down rapidly. ${ }^{23} \mathrm{~A}$ buildup of carriers at the band gap reduces the band gap absorption (absorption bleach, AB), as can be seen in Figure 1 . Here, a bleach is indicated by a positive value for $\Delta \alpha=$ $\alpha_{0}-\alpha$, where $\alpha_{0}$ is the steady state absorbance and $\alpha$ is the absorbance after the pump pulse. However, below the band gap (1700 to $2000 \mathrm{~nm}$ ), we observe a negative $\Delta \alpha$ for both materials, indicating photoinduced absorption (PA)

To gain insight into the nature and strength of the $\mathrm{PA}$, we performed power and wavelength-dependent measurements. At high fluences, when more than one exciton is created per QD, the distribution of multiexcitons is Poissonian, and the decay of the TA signal is governed by Auger recombination. ${ }^{24}$ After 500 ps, all multiexcitons have recombined, leaving a population of singly excited QDs. Assuming an initial Poissonian distribution of carriers, the normalized differential absorption after 500 ps becomes

$$
\frac{\Delta \alpha_{500 \mathrm{ps}}}{\alpha_{0}}=\frac{2}{g}\left(1-e^{-N_{\mathrm{eh}}}\right)
$$

Here we have expressed the pump power as the average number $N_{\text {eh }}$ of excitons that are created per QD by the pump pulse (see Supporting Information). The value of $g$ is the degeneracy of the LUMO and the HOMO. The factor 2 accounts for both electrons and holes since the differential absorption is proportional to the sum of all involved carriers. ${ }^{25}$

The power dependence of the PA for short and long times after the pump pulse is given in Figure 1 for $\mathrm{PbSe}$. A similar behavior is observed for $\mathrm{PbSe} / \mathrm{CdSe}$ (see Figure S-1 in Supporting Information). At short delay times (red curves), the PA is linear with $N_{\text {eh }}$ within the given power range, in agreement with a Poisson distribution of the initial excitons. At long times, we observe a trend, which fits well to eq 1 .

On the basis of these traces, we quantify the strength of the $A B$ and $P A$ transition as the absorbance per exciton $\alpha_{A B, \text { ex }}$ and $\alpha_{P A, \text { ex }}$ respectively; $\alpha_{P A, \text { ex }}$ is the maximum PA after long delay, obtained from fitting eq 1 to the black curves in Figure 2 (see Table 1). For PbSe, $\alpha_{A B, e x}$ (obtained in the same way) is $16.9 \times$ $10^{-3} \mathrm{~cm}^{-1}$ ( $\mathrm{fwhm}=81 \mathrm{meV}$ ). The intraband transition is about 10 times weaker. The PA per exciton $\alpha_{\mathrm{PA}, \mathrm{ex}}$ in $\mathrm{PbSe} / \mathrm{CdSe}$ is also about 10 times weaker than the $\alpha_{A B, e x}\left(88 \times 10^{-4} \mathrm{~cm}^{-1}\right.$, fwhm $\left.=105 \mathrm{meV}\right)$. To compare both materials, the absorption coefficient $\mu^{26}$ and the cross section $\sigma=\mu \mathrm{V}_{\mathrm{QD}}$ for the PA are also given in Table 1 . The cross sections are very similar for both materials. The larger volume of $\mathrm{PbSe} / \mathrm{CdSe}$ results in a lower $\mu$ than $\mathrm{PbSe}$ QDs with similar core size. The $\mu$ is proportional to the oscillator strength and the local field factor. ${ }^{27}$ Given the increasing local field factor in core/shell QDs compared to core only QDs, ${ }^{28}$ a lower 


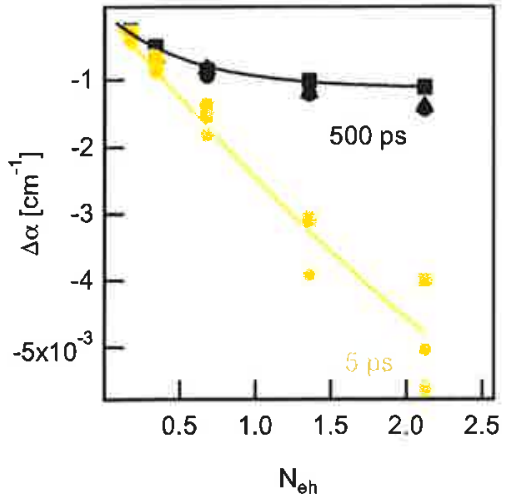

Figure 2. Differential absorption as a function of average number of excitons $N_{\text {eh }} 5$ ps after the pump pulse (orange) and $500 \mathrm{ps}$ after the pump pulse (black) at $1700 \mathrm{~nm}$ (triangles), $1850 \mathrm{~nm}$ (squares), and $2000 \mathrm{~nm}$ (circles) for PbSe QDs. The solid lines are fits to a Poissonian state filling model.

TABLE 1. Photoinduced Absorbance for a Population of One Exciton in the Initial State $\alpha_{\mathrm{PA}, \mathrm{ex}}$, Derived from a Poissonian Fit, Together with the Absorption Coefficient for PbSe and PbSe/CdSe QDs

\begin{tabular}{|c|c|c|c|c|c|c|}
\hline \multirow[b]{2}{*}{$\begin{array}{l}\lambda_{\text {probe }} \\
(\mathrm{nm})\end{array}$} & \multicolumn{3}{|c|}{ PbSe } & \multicolumn{3}{|c|}{$\mathrm{PbSe} / \mathrm{CdSe}$} \\
\hline & $\begin{array}{l}\alpha_{\mathrm{PA}, \mathrm{ex}} \\
\left(\mathrm{cm}^{-1}\right)\end{array}$ & $\begin{array}{c}\mu_{\mathrm{P}, e \mathrm{ex}} \\
\left(\mathrm{mm}^{-1}\right)\end{array}$ & $\begin{array}{l}\sigma_{\mathrm{PA}, \mathrm{ex}} \\
\left(\mathrm{m}^{2}\right)\end{array}$ & $\begin{array}{c}\alpha_{\mathrm{PA}, e x} \\
\left(\mathrm{~cm}^{-1}\right)\end{array}$ & $\begin{array}{l}\mu_{\mathrm{PA}_{\mathrm{A}, \mathrm{ex}}} \\
\left(\mathrm{cm}^{-1}\right)\end{array}$ & $\begin{array}{l}\sigma_{\mathrm{PA}, \mathrm{ex}} \\
\left(\mathrm{m}^{2}\right)\end{array}$ \\
\hline 1700 & $1.54 \times 10^{-3}$ & $\begin{array}{ll}3 & 187\end{array}$ & $9.2 \times 10^{-18}$ & $8.6 \times 10^{-4}$ & +92 & $8.0 \times 10^{-18}$ \\
\hline 1850 & $1.29 \times 10^{-3}$ & 157 & $7.7 \times 10^{-18}$ & $5.6 \times 10^{-4}$ & 82 & $7.1 \times 10^{-18}$ \\
\hline 2000 & $1.57 \times 10^{-3}$ & 190 & $9.7 \times 10^{-18}$ & $8.6 \times 10^{-4}$ & 92 & $8.0 \times 10^{-18}$ \\
\hline
\end{tabular}

$\mu$ translates in a lower oscillator strength for $\mathrm{PbSe} / \mathrm{CdSe}$ QDs.

Figure 3 shows that after 10 ps delay the transient absorption for $\mathrm{PbSe}$ at the band gap maximum (red trace) and below the band gap (black traces, inverted for clarity) follows a very similar time dependence for three different values of the pump power. This decay is due to Auger recombination. Fitting a sum of three exponentials to the absorption transients (see Supporting Information about the fitting procedure) yields a biexciton lifetime of $82 \mathrm{ps}$, a triexciton lifetime of $32 \mathrm{ps}$, and a quadrexciton lifetime of $13 \mathrm{ps}$ for the PbSe QDs at the band gap. The PA transients differ from the transients at the band gap for early delay times. The onset is much steeper, which is clearest for the highest pump power (see Figure 3c). For both high and low pump powers, an initial decay is present, which we do not observe in the differential absorption transients around the band gap. The fitting procedure (see Supporting Information) yields a lifetime of $1.2 \mathrm{ps}$ for this initial decay. Similar dynamics are observed for $\mathrm{PbSe} / \mathrm{CdSe} \mathrm{QDs}$, which is summarized in Table 2.

TABLE 2. Auger Decay Times and Initial Decay Times for PbSe and PbSe/CdSe QDs, As Extracted by the Fitting Proceduce

\begin{tabular}{lcc} 
[ps] & PbSe & PbSe/CdSe \\
\hline$\tau_{\mathrm{A}, \mathrm{n}=2}$ & $82 \pm 7.7$ & $84 \pm 6.4$ \\
$\tau_{\mathrm{A}, \mathrm{n}=3}$ & $32 \pm 8.9$ & $16 \pm 0.84$ \\
$\tau_{\mathrm{A}, \mathrm{n}=4}$ & $13 \pm 3.5$ & \\
$\tau_{\text {hor }}$ & $1.2 \pm 0.13$ & $1.3 \pm 0.24$
\end{tabular}

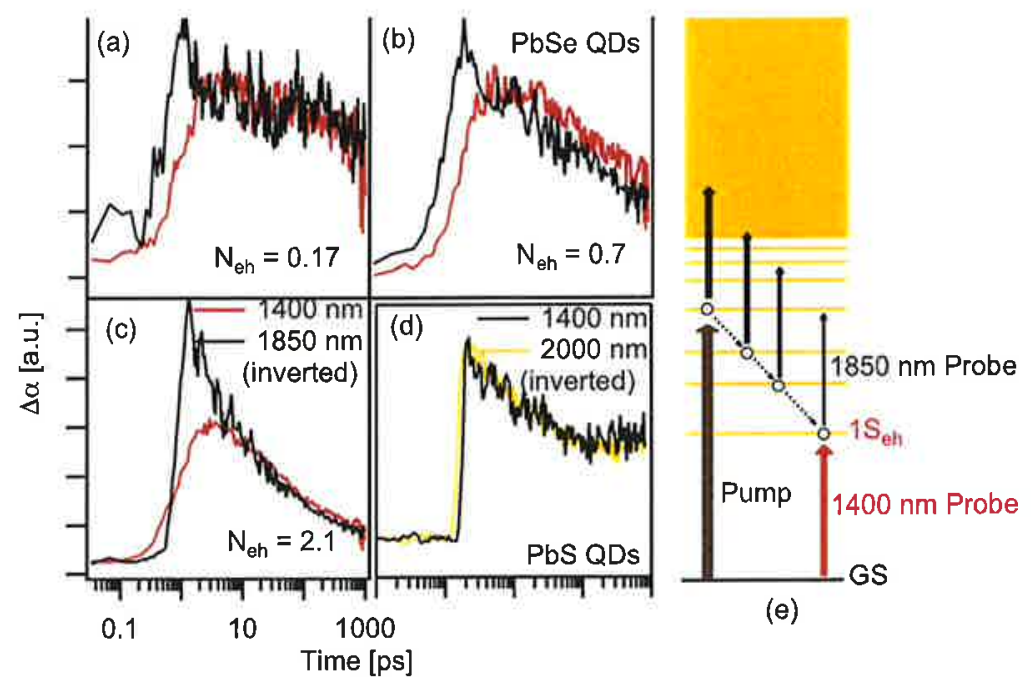

Figure 3. (a-c) Absorption transients for the differential absorption at the band gap ( $1450 \mathrm{~nm}$, red) and below the band gap (1850 nm, black, inverted for clarity) for PbSe QDs show similar decay dynamics after 10 ps for three different values of the pump power: (a) $N_{\mathrm{eh}}=0.17$, (b) $N_{\mathrm{eh}}=0.7$, and (c) $N_{\mathrm{eh}}=2.1$. An initial faster decay is present in the PA transients for all three pump powers shown. (d) Absorption transients for very small PbS QDs ( $2.45 \mathrm{~nm}$ ) for which the pump is resonant with the band gap transition $(780 \mathrm{~nm})$ showing PA $(\Delta \alpha<0)$, yet no fast initial decay component. (e) Intraband transitions probed by the subgap probe (black). These transitions cause the fast initial decay component in the subgap transients (black) for nonresonantly excited $\mathrm{PbSe} \mathrm{QDs}$. The band gap probe (red) will only register the band gap bleach after carriers have cooled to the band gap. 
The band gap bleach only develops when hot carriers have cooled to the band gap. However, the fast rise of the PA suggests that hot carriers also exhibit PA (see Figure $3 \mathrm{~b}$ ). To corroborate this, we measured the TA of very small PbS QDs $(2.45 \mathrm{~nm})$, for which the pump is resonant with the band gap transition (see Figure $3 b$ ). In this case, no fast initial decay component is observed, which corroborates the assumption that hot electrons and holes also show PA.

\section{DISCUSSION}

The wavelength-independent PA we observe in these near-infrared QDs could have different origins, such as (1) Coulomb shifts in the multiexcitonic absorption spectrum, ${ }^{29-34}$ (2) absorption from surfacelocalized states or defects, ${ }^{14,35-37}$ or (3) intraband absorption. ${ }^{6,9,15,38}$ Coulomb shifts can be excluded since the PA is observed up to $2000 \mathrm{~nm}$, well below the band gap. Moreover, a Coulomb shift would result in a characteristic antisymmetric TA spectrum, ${ }^{25}$ not the broadband signal we observe here (see Figure 5-3a).

Malko et al. ${ }^{12}$ reported PA in absorption transients of colloidal CdSe QDs. An extensive study revealed a strong dependence on solvent and surface passivation, along with a limited size dependence of the PA cross section. This study indicates a strong influence of the QD surface. Given the high cross section, Malko et al. attributed the TA to transitions from dangling surface bonds to excited states in the capping or surrounding matrix, rather than intraband absorption. Since we work with a considerably lower photon energy $(0.5$ to $0.8 \mathrm{eV}$ vs 2.0 to $2.5 \mathrm{eV}$ ), a transition to a final state in the surrounding matrix is unlikely at these low energies. Furthermore, since the dynamics and strength of the $\mathrm{PA}$ of $\mathrm{PbSe}$ and $\mathrm{PbSe} / \mathrm{CdSe} \mathrm{QDs}$ are very similar, despite having very distinct surface properties, this makes PA from surface defects improbable. Moreover, the PA we observe at long delay times saturates with increasing pump power, similar to the bleach of the band gap absorption. This shows that the initial state of the PA after carrier cooling is in fact the band gap exciton. That QD core states are involved, instead of surface states, is further confirmed by the Auger dynamics of the PA signal. We therefore conclude that the PA observed here finds its origin in intraband absorption. This is in line with a recent study of photocharging ${ }^{39,40}$ of $\mathrm{PbSe}$ QDs, where pumping with $1.5 \mathrm{eV}$ photons did not result in significant photocharging. On the basis of the interpretation of the PA as intraband absorption, we attribute the quick rise time to intraband transitions from higher energy excited states (see Figure 3a). The correspondence between the time constant of the initial decay (see Table 2) we measure and literature values for intraband carrier cooling ${ }^{4,5,41}$ corroborates this interpretation.

Intraband absorption is forbidden in first order for bulk semiconductors. In order to conserve the
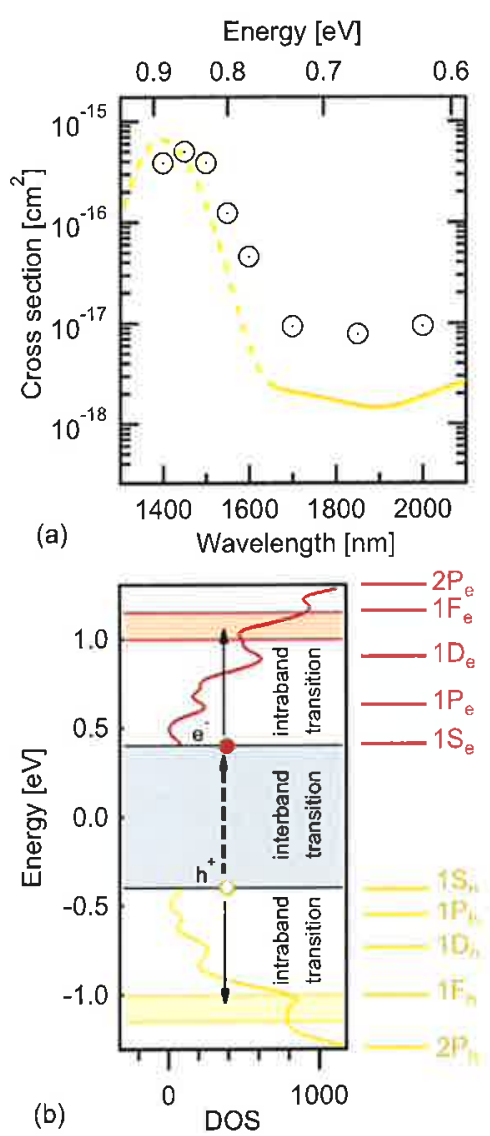

Figure 4. (a) Absorption cross section in the presence of an exciton for PbSe QDs $(4.5 \mathrm{~nm}$ ) as calculated by tight-binding calculations (red trace) agrees well qualitatively with the values measured using TA spectroscopy (black circles). (b) Density of states for PbSe QDs (4.5 nm) as calculated by tight-binding calculations illustrates the transitions that give the strongest contribution to the absorption cross section in the presence of an exciton for energies below the band gap (solid line arrow) and at the band gap (dashed line arrow). The same symbolism is used in (a).

crystal momentum, phonon absorption or emission is needed. The process is commonly called free carrier absorption (FCA) and is well described by a Drude model. In the case of a particle in an infinite spherical potential well, only transitions with orbital angular momentum $\Delta l= \pm 1$ are allowed. Here, we find that both the conservation of crystal momentum and orbital angular momentum are relaxed in $\mathrm{Pb}$ chalcogenide QDs, resulting in a nonzero probability for intraband absorption. This confirms that in quantum dots the deviations from the ideal infinite spherical potential well cause the electronic states to be superpositions of states with different crystal momentum and orbital angular momentum. ${ }^{10}$

Tight-Binding Calculation. Tight-binding calculations $\mathrm{s}^{20}$ on $4.5 \mathrm{~nm}$ PbSe QDs support our observations. We simulate the spectrum of the absorption cross section without and with an exciton in the HOMO-LUMO. Our simulations substantiate earlier atomistic pseudopotential calculations $s^{42}$ of the $A B$ at the band gap and 


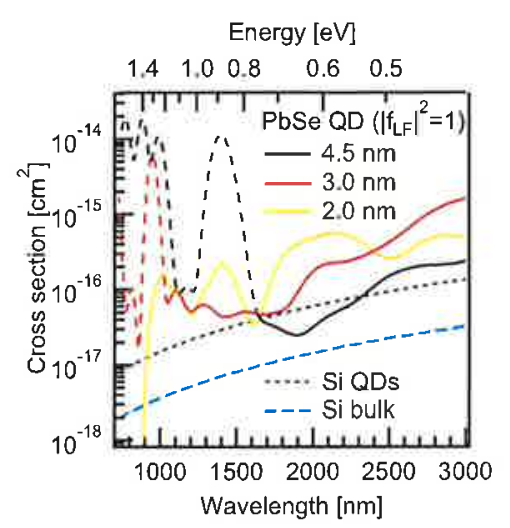

Figure 5. Absorption cross section in the presence of an exciton for PbSe QDs (black, $d=4.5 \mathrm{~nm}, E_{\mathrm{g}}=0.89 \mathrm{eV}$; red, $d=$ $3.0 \mathrm{~nm}, E_{\mathrm{g}}=1.3 \mathrm{eV}$; orange, $d=2.0 \mathrm{~nm}, E_{\mathrm{g}}=1.92 \mathrm{eV}$ ) as calculated by tight-binding calculations, taking no local field factor into account, shows size-dependent intraband absorption that cannot be explained by a simple Drude model $\left(\sim \lambda^{2}\right)$. The cross section dominated by intraband transitions is marked with a solid line, whereas the cross section dominated by interband transitions is marked by a dashed line. For comparison with materials which operate in the same wavelength range, the absorption cross section for FCA in bulk silicon ${ }^{45}$ (blue stripes) and silicon nanocrystals ${ }^{47}$ (brown dots) is shown.

intraband absorption between the 15 and $1 \mathrm{P}$ state $\mathrm{e}^{10}$ (see Figure S-4a). Moreover, they predict previously unreported nonzero oscillator strength for intraband transitions between the HOMO-LUMO and higher energy states, in the energy range between the 1S-to-1P and the band gap transition (see Figure 4b). Our experimental data qualitatively agree with the tight-binding results, taking the local field factor $\left(\left|f_{\mathrm{LF}}\right|^{2}=0.059\right)^{28,43}$ in $\mathrm{C}_{2} \mathrm{Cl}_{4}$ into account (see Figure 4a). Uncertainty of the local field factor or excitonic effects which are missing in the calculations could explain the difference between theory and experiment. The final states of the intraband transitions probed in this work are quasicontinuum states, as illustrated in the density of states diagram in Figure $4 \mathrm{~b}$, with a mixed $1 \mathrm{~F}$ and $2 \mathrm{P}$ character. Electron and hole transitions contribute equally to the cross section (see Figure $5-4 b$ ). This is in line with the similar bulk effective masses but contradicts the prediction of a denser hole manifold. ${ }^{10}$ Tight-binding calculations also predict a higher intraband absorption cross section for carriers in the higher energy $P$ state (see Figure S-5). This confirms our interpretation of the quick rise time and fast initial decay (see Figure $3 a-c$ and Table 2) as intraband absorption from carriers cooling to the band gap. Similar cross sections are found for intraband transitions directly below the band gap for $4.5 \mathrm{~nm}$ wurtzite CdSe QDs (see Figure S-6b). However, the predicted ratio between $A B$ and $P A$, due in part to the lower degeneracy of HOMO-LUMO in CdSe, is much higher ( $\sim 400)$, making it harder to distinguish the PA in a TA experiment. Moreover, the reported cross sections for PA in CdSe are much higher than the theoretical values predicted by tight-binding calculations.
This highlights the clear difference between the PA in $\mathrm{CdSe}$ as reported in the literature, which can be attributed to nanocrystal charging and the PA in PbSe reported here, which is due to intraband transitions.

Benchmarking with Silicon. FCA and FCR (free carrier refraction) in silicon have been studied extensively for applications in ultrafast signal processing at telecom wavelengths. ${ }^{44}$ In bulk silicon, FCA is well understood and its cross section has a $\lambda^{2}$ dependence in line with the predictions of the Drude model. ${ }^{44,45}$ Silicon nanocrystals (NCs, $d=5 \mathrm{~nm}$ ) in silicon-rich oxide, which operate in the same wavelength range, were shown to have an order of magnitude higher cross sections for FCA, both in the visible ${ }^{46}$ and in the near-infrared ${ }^{47}$ region, yet with a similar $\lambda^{2}$ dependence. The result was explained by considering that the mean free path of the carriers was limited to the QD size, increasing the scattering cross section.

In Figure 5, we compare the FCA cross section of bulk Si and Si NCs with the calculated spectrum of the absorption cross section of excited PbSe QDs of three different sizes. Since a typical application will require a densely packed layer of QDs, we do not take the local field factor into account $\left(\left|f_{\mathrm{LF}}\right|^{2}=1\right)$. Depending on the QD diameter, the intraband absorption cross section is $1-2$ orders of magnitude larger than in bulk Si. Around $1550 \mathrm{~nm}$, an important telecom wavelength, the cross section is comparable to the Si NC cross section. As the origin of the absorption is a direct intraband absorption, without interaction of phonons, the wavelength dependence differs from a Drude model and strongly depends on the QD diameter.

As highlighted by Kekatpure and Brongersma, ${ }^{46}$ the higher FCA cross section has important implications for gain at telecom wavelengths using Si NCs in erbiumdoped silica. For the erbium gain to exceed the FCA loss, FCA cannot be larger than $10^{-18} \mathrm{~cm}^{2}$. A similar upper limit to the photoinduced loss of QDs doped with erbium exists, making gain in hybrid erbium - QD systems impossible. The use of QDs directly for gain will be affected, as well. Assuming a similar ratio between the gain cross section and the intraband absorption cross section at the band gap as the ratio $\alpha_{\mathrm{AB}, \mathrm{ex}} / \alpha_{\mathrm{PA}, \mathrm{ex}} \simeq 10$ we observed experimentally, the theoretical threshold for gain would increase by about $11 \%$ to $N_{\text {eh }}=4.45$. Similarly, the maximum gain value will decrease by $20 \%$ (see Supporting Information). Note that in colloidal QDs the rate of multiexciton decay (a necessary condition to obtain population inversion) is dominated by the nonradiative Auger recombination rate, which is $4-6$ orders of magnitude larger than the spontaneous emission recombination rate. ${ }^{48}$ Therefore, a high QD density and large total number of QDs coupled to the laser cavity will be required for the stimulated emission rate to overcome the nonradiative recombination rate. ${ }^{49}$ In turn, this requires very high pump fluences, essentially making $\mathrm{CW}$ lasing impossible. Moreover, in the case of 
nonresonant excitation, the Auger recombination rate for $N_{\text {eh }} \simeq 4$ approaches the rate of carrier cooling to the band gap. This inhibits gain altogether, as the condition of population inversion is never reached.

Intraband Absorption for Integrated Optical Modulators. The intraband absorption observed in lead salt colloidal QDs can be beneficial for other applications. Siliconbased electro-optical modulators typically do not use FCA since the effect is too weak to make small modulators with easily achievable doping levels. Modulators are then based on free carrier refraction (FCR), where a trade-off is made between an optimized modulation depth and minimal insertion loss because of FCA. Moreover, the phase shift that FCR induces requires these type of modulators to have an interferometric design, which limits the spectral bandwidth at which they can be used. The intraband absorption of lead salt colloidal QDs is a first step toward a zeroinsertion loss optical modulator. The small size, together with the high intraband absorption cross section, makes high absorption coefficients on the order of a few 100 to $1000 \mathrm{~cm}^{-1}$ easily achievable with doping or electrical injection. ${ }^{50}$ Modulators with footprints between 10 and $100 \mu \mathrm{m}$, an order of magnitude smaller than current interferometric designs, are therefore possible. Moreover, the extremely large absorption cross section at and above the band gap (see Figure $4 a$ ), together with the tunability of band gap with size, makes optical excitation of the intraband absorption, as demonstrated here, very easy. It opens the way for all optical signal processing using lead salt QDs.

\section{CONCLUSION}

In conclusion, we have shown that intraband absorption in the telecom wavelength range is universal to lead chalcogenide QDs. The absorption is strong, only 10 times weaker than the band gap absorption bleach. The intraband absorption is broadband and nearly constant over a large wavelength range. This range can be tuned easily by changing the QD size. The intraband absorption has ultrafast dynamics in the one to tens of picoseconds range, governed by carrier cooling and Auger recombination. Tight-binding modeling of the absorption cross section of excited QDs confirms our experimental observation of intraband absorption in this wavelength range.

Our work is essential to understand the response of QDs as gain media and ultrafast nonlinear medic for photonic applications. Moreover, our measurements are a clear manifestation that the selection rules for the bulk crystal momentum and for the angular momentum in spherically confined structures as mentioned above are relaxed in QDs and are therefore very interesting from a theoretical standpoint. They go beyond inter-sub-band absorption ${ }^{6-9,11}$ and cover a new wavelength range directly below the band gap. Intraband absorption offers a nice way to study the dynamics of carrier cooling $^{3-5}$ and multiple exciton generation ${ }^{51,52}$ in a direct way, free from possible Coulomb shifts or redistribution over dark and bright band gap states, which complicate typical TA measurements at the band gap.

\section{METHODS}

Materials. Technical oleic acid (90\%) was purchased from Sigma-Aldrich. $\mathrm{PbCl}_{2}$ (99.999\%), $\mathrm{PbO}$ (99.999\%), and selenium powder ( -200 mesh, $99.999 \%$ ) were ordered from Alfa-Aesar. CdO (99.999\%) and tri-n-octylphosphine $(97 \%)$ were obtained from Strem Chemicals. Diphenyl ether, methanol, butanol, and toluene were all of quality "for synthesis" and were ordered from VWR.

PbSe Synthesis. Monodisperse colloidal PbSe QDs were synthesized by a high-temperature synthesis developed by Murray et al. ${ }^{53}$ The ligand shell consists of oleic acid (OA), as shown by nuclear magnetic resonance spectroscopy. ${ }^{54}$

$\mathrm{PbSe} / \mathrm{CdSe}$ Cation Exchange. PbSe/CdSe core-shell QDs were synthesized starting from $\mathrm{PbSe}$ core $\mathrm{QDs}$ by means of a cation exchange reaction. ${ }^{55}$ In this reaction, $\mathrm{Cd}$-oleate $(0.3 \mathrm{M}$ stock solution) is added to a PbSe QD suspension in toluene at $100^{\circ} \mathrm{C}$ (the typical $\mathrm{Pb} / \mathrm{Cd}$ ratio used is $1: 10$ ). After $40 \mathrm{~min}$, the reaction is quenched using a mixture of $\mathrm{MeOH}$ and $\mathrm{BuOH}(1: 2)$ and precipitated twice.

Femtosecond Transient Absorption Spectroscopy. The QDs were dispersed in tetrachloroethylene for the measurements. The sample is carefully kept free from oxygen and water contam ination, both during its preparation and during the measurement. Time- and energy-resolved transient absorption spectroscopy was performed using a commercial Ti:S laser system (Mira- Legend USP, Coherent Inc.) and optical parametric amplification for both the pump (Topas-800-fs, Coherent Inc.) and probe beams (Opera, Coherent Inc.). Pump and probe beams overlapped under a small angle $\left(3^{\circ}\right)$ in a cuvette of $10 \mathrm{~mm}$ path length and were imaged onto InGaAs pin photodiodes (Hamamatsu G5853-23, G8605-23). The polarization of the pump and probe was made orthogonal so that pulses of similar photon energy could be separated with polarization filters. The experimental setup permits variation in the delay time between the pump and probe pulses in a tempora window extending to $1 \mathrm{~ns}$. The measured fractional transmis sion signals, called transient absorption (TA) in the following, may be written as TA $=\left(T_{\text {on }}-T_{\text {off }}\right) / T_{\text {off }}$, where $T_{\text {on }}$ denotes the probe transmission with pump on and $T_{\text {off }}$ the probe transmission with pump off. With this definition, bleach and emission yield positive TA signals while an increase in absorption yields negative TA signals.

Tight-Binding Calculation. The single-electron states and energies of PbSe QDs are calculated using the $s \mathrm{p}^{3} \mathrm{~d}^{5} \mathrm{~s}^{*}$ tight-binding model of Allan and Delerue ${ }^{20}$ confinement including spin-orbit coupling. Since many states are needed to calculate the optica spectra, the matrix of the tight-binding Hamiltonian is fully diagonalized even for diameters up to $4.5 \mathrm{~nm}(\approx 1600$ atoms) For more information, refer to the Supporting Information.

Conflict of interest: The authors declare no competing financial interest.

Acknowledgment. This work is part of the Joint Solar Pro gramme (JSP) of the Stichting voor Fundamenteel Onderzoek der Materie FOM, which is supported financially by the Nederlandse Organisatie voor Wetenschappelijk Onderzoek (NWO). This work is co-financed by Nuon Helianthos. This work has also 
been financially supported by the 3TU Centre for Sustainable Energy Technologies (Federation of the Three Universities of Technology in The Netherlands). The authors acknowledge BelSPo (IAP 6.10, photonics@be), EU-FP7 (Navolchi), and Ghent University for funding. B.D.G. acknowledges financial support by the Bijzonder Onderzoeksfonds of Ghent University. A.J.H. acknowledges financial support by The Netherlands Organisation for Scientific Research (NWO) through a NWO-VEN grant. D.V.T. acknowledges the ERC for an ERC Starting Grant (ULPPIC).

Supporting Information Available: Results on $\mathrm{PbSe} / \mathrm{CdSe}$ QDs, fitting procedures used, more information, and results from tight-binding simulations and estimation of gain including intraband absorption. This material is available free of charge via the Internet at http://pubs.acs.org.

\section{REFERENCES AND NOTES}

1. Sargent, E. Infrared Quantum Dots. Adv. Mater. 2005, 17 , 515-522.

2. Lifshitz, E.; Brumer, M.; Kigel, A.; Sashchiuk, A.; Bashouti, M.; Sirota, M.; Galun, E.; Burshtein, Z.; Le Quang, A. Q.; Ledoux Rak, l.; et al. Stable PbSe/PbS and $\mathrm{PbSe} / \mathrm{PbSe}_{x} \mathrm{~S}_{1-x}$ CoreShell Nanocrystal Quantum Dots and Their Applications. J. Phys. Chem. B 2006, 110, 25356-25365.

3. Schins, J. M.; Trinh, M. T.; Houtepen, A. J.; Siebbeles, L. D. A. Probing Formally Forbidden Optical Transitions in PbSe Nanocrystals by Time- and Energy-Resolved Transien Absorption Spectroscopy. Phys. Rev. B 2009, 80, 035323.

4. An, J. M.; Califano, M.; Franceschetti, A.; Zunger, A. ExcitedState Relaxation in PbSe Quantum Dots. J. Chem. Phys. 2008, 128, 164720

5. Harbold, J. M.; Wise, F. W. Photoluminescence Spectroscopy of PbSe Nanocrystals. Phys. Rev. B 2007, 76, 125304

6. Wehrenberg, B.; Wang, C.; Guyot-Sionnest, P. Interband and Intraband Optical Studies of PbSe Colloidal Quantum Dots. J. Phys. Chem. B 2002, 106, 10634-10640.

7. Guyot-Sionnest, P.; Hines, M. Intraband Transitions in Semiconductor Nanocrystals. Appl. Phys. Lett. 1998, 72, 686-688.

8. Wang, C; Shim, M.; Guyot-Sionnest, P. Electrochromic Nanocrystal Quantum Dots. Science 2001, 291, 2390-2392.

9. Pandey, A.: Guyot-Sionnest, P. Intraband Spectroscopy and Band Offsets of Colloidal II-VI Core/Shell Structures. J. Chem. Phys. 2007, 127, 104710

10. An, J. M.; Franceschetti, A.; Dudiy, 5. V.; Zunger, A. The Peculiar Electronic Structure of PbSe Quantum Dots. Nano Lett. 2006, 6, 2728-2735.

11. Yang, $Y_{, i}$ Rodríguez-Córdoba, $W_{i ;}$ Lian, T. Ultrafast Charge Separation and Recombination Dynamics in Lead Sulfide Quantum Dot-Methylene Blue Complexes Probed by Electron and Hole Intraband Transitions. J. Am. Chem. Soc. 2011, 133, 9246-9249.

12. Malko, A.; Mikhailovsky, A.; Petruska, M.; Hollingsworth, J.; Htoon, $H_{\text {.; }}$ Bawendi, M.; Klimov, V. From Amplified Spontaneous Emission to Microring Lasing Using Nanocrysta Quantum Dot Solids. Appl. Phys. Lett. 2002, 81, 1303-1305.

13. Cret, A.; Zavelani-Rossi, M.; Lanzani, G.; Anni, M.; Manna, L, Lomascolo, M. Role of the Shell Thickness in Stimulated Emission and Photoinduced Absorption in CdSe Core/ Shell Nanorods. Phys. Rev. B 2006, 73, 165410.

14. Sewall, S. L.; Cooney, R. R.; Anderson, K. E. H.; Dias, E. A. Sagar, D. M.; Kambhampati, P. State-Resolved Studies of Biexcitons and Surface Trapping Dynamics in Semiconductor Quantum Dots. J. Chem. Phys. 2008, 129, 084701.

15. Ginger, D. S.; Ginger, D.; Dhoot, A. S.; Dhoot, A.; Finlayson, C. E.; Finlayson, C.; Greenham, N. C.; Greenham, N. LongLived Quantum-Confined Infrared Transitions in CdSe Nanocrystals. Appl. Phys. Lett. 2000,77.

16. Huang, J.; Huang, Z.; Jin, S.; Lian, T. Exciton Dissociation in CdSe Quantum Dots by Hole Transfer to Phenothiazine. J. Phys. Chem. C 2008, 112, 19734-19738.

17. Zhu, H.; Song, N.; Rodríguez-Córdoba, W.; Lian, T. Wave Function Engineering for Efficient Extraction of up to Nineteen Electrons from One CdSe/CdS Quasi-Type Quantum Dot. J. Am. Chem. Soc. 2012, 134, 4250-4257.
18. Leventis, H. C. O'Mahony, F. Akhtar, J; Afzaal, M: O'Brien, P.; Haque, S. A. Transient Optical Studies of Interfacia Charge Transfer at Nanostructured Metal Oxide/PbS Quantum Dot/Organic Hole Conductor Heterojunctions. J. Am. Chem. Soc. 2010, 132, 2743-2750

19. Yang, Y.; Rodríguez-Córdoba, W.; Xiang, X.; Lian, T. Strong Electronic Coupling and Ultrafast Electron Transfer between $\mathrm{PbS}$ Quantum Dots and $\mathrm{TiO}_{2}$ Nanocrystalline Films. Nano Lett. 2012, 12, 303-309.

20. Allan, G.; Delerue, C. Confinement Effects in PbSe Quantum Wells and Nanocrystals. Phys. Rev. B 2004, 70, 245321

21. Thomson, D; Gardes, F.; Fedeli, J.-M.; Zlatanovic, S.; Hu, Y. Kuo, B.; Myslivets, E.; Alic, N.; Radic, S.; Mashanovich, G.; et al. 50-Gb/S Silicon Optical Modulator. IEEE Photonics Technol. Lett. 2012, 24, 234-236.

22. Liu, A.; Jones, R.; Liao, L.; Samara-Rubio, D.; Rubin, D.; Cohen, O.; Nicolaescu, R.; Paniccia, M. A High-Speed Silicon Optical Modulator Based on a Metal-Oxide-Semiconductor Capacitor. Nature 2004, 427, 615-618.

23. Klimov, V.; McBranch, D. Femtosecond High-Sensitivity Chirp-Free Transient Absorption Spectroscopy Using Kilohertz Lasers. Opt. Lett. 1998, 23, 277-279.

24. Klimov, V.; Mikhailovsky, A.; McBranch, D.; Leatherdale, C. Bawendi, M. Quantization of Multiparticle Auger Rates in Semiconductor Quantum Dots. Science 2000, 287, $1011-$ 1013.

25. Trinh, M. T.: Houtepen, A. J: Schins, J. M.; Piris, J:; Siebbeles, L. D. A. Nature of the Second Optical Transition in PbSe Nanocrystals. Nano Lett. 2008, 8, 2112-2117.

26. Moreels, I.; Hens, Z. On the Interpretation of Colloidal Quantum-Dot Absorption Spectra. Small 2008, 4, 1866-1868.

27. De Geyter, B.; Justo, Y.; Moreels, I.; Lambert, K.; Smet, P. F.; Van Thourhout, D.; Houtepen, A. J.; Grodzinska, D.; de Mello Donega, C.; Meijerink, A.; et al. The Different Nature of Band Edge Absorption and Emission in Colloidal PbSe/ CdSe Core/Shell Quantum Dots. ACS Nano 2011, 5, 58-66.

28. De Geyter, B.; Hens, Z. The Absorption Coefficient of PbSe/ CdSe Core/Shell Colloidal Quantum Dots. Appl. Phys. Lett. 2010, 97, 161908.

29. Hwang, Y.-N.: Je, K.-C.: Kim, D.: Park, S.-H. Observation of Enhanced Biexcitonic Effect in Semiconductor Nanocrystals. Phys. Rev. B 2001, 64, 041305.

30. Hu, Y. Z.; Koch, S. W.; Lindberg, M.; Peyghambarian, N.; Pollock, E. L.; Abraham, F. F. Biexcitons in Semiconductor Quantum Dots. Phys. Rev. Lett. 1990, 64, 1805-1807.

31. Kang, K. I; Kepner, A. D.; Gaponenko, S. V; Koch, S. W.; Hu Y. Z.; Peyghambarian, N. Confinement-Enhanced Biexciton Binding Energy in Semiconductor Quantum Dots. Phys. Rev. B 1993, 48, 15449-15452.

32. Woggon, $\mathrm{U}_{\text {; }}$ Giessen, $\mathrm{H}_{\text {; }}$ Gindele, F,; Wind, O.; Fluegel, $\mathrm{B}$ Peyghambarian, N. Ultrafast Energy Relaxation in Quantum Dots. Phys. Rev. B 1996, 54, 17681-17690.

33. Klimov, $V_{;}$Hunsche, $S_{i}$ Kurz, H. Biexciton Effects in Femtosecond Nonlinear Transmission of Semiconductor Quantum Dots. Phys. Rev. B 1994, 50, 8110-8113.

34. Gesuele, F.; Sfeir, M; Koh, W.-k,i Murray, C. B;; Heinz, T. F Wong, C. W. Ultrafast Supercontinuum Spectroscopy of Carrier Multiplication and Biexcitonic Effects in Excited States of PbS Quantum Dots. Nano Lett. 2012, 12, 26582664.

35. Malko, A.; Mikhailovsky, A.; Petruska, M.; Hollingsworth, J. Klimov, V. Interplay between Optical Gain and Photoinduced Absorption in CdSe Nanocrystals. J. Phys. Chem. 8 2004, 108, 5250-5255.

36. Zhang, J.; Jiang, X. Steady State Photoinduced Absorption of PbS Quantum Dots Film. Appl. Phys. Lett. 2008, 92, 141108.

37. Cooney, R.; Sewall, S.; Sagar, D.; Kambhampati, P. Gain Control in Semiconductor Quantum Dots via StateResolved Optical Pumping. Phys. Rev. Lett. 2009, 102, 127404

38. Qu, Y; Jia, W.; Zheng, Y.; Ying, J. Y. Auger Recombination and Intraband Absorption of Two-Photon-Excited Carriers in Colloidal CdSe Quantum Dots. Appl. Phys. Lett. 2007, 90, 133112

39. McGuire, J. A.; Sykora, M.; Robel, I.; Padilha, L. A.; Joo, J.; Pietryga, J. M.; Klimov, V. I. Spectroscopic Signatures of 
Photocharging Due to Hot-Carrier Transfer in Solutions of Semiconductor Nanocrystals under Low-Intensity Ultraviolet Excitation. ACS Nano 2010, 4, 6087-6097.

40. Padilha, L. A.; Robel, I.; Lee, D. C.; Nagpal, P.; Pietryga, J. M. Klimov, V. I. Spectral Dependence of Nanocrystal Photoionization Probability: The Role of Hot-Carrier Transfer. ACS Nano 2011, 5, 5045-5055.

41. Schaller, R.; Pietryga, J.; Goupalov, S.; Petruska, M.; Ivanov, S. Klimov, V. Breaking the Phonon Bottleneck in Semiconductor Nanocrystals via Multiphonon Emission Induced by Intrinsic Nonadiabatic Interactions. Phys. Rev. Lett. 2005, 95, 196401

42. An, J. M.; Franceschetti, A.; Zunger, A. Pauli Blocking versus Electrostatic Attenuation of Optical Transition Intensities in Charged PbSe Quantum Dots. Phys. Rev. $B$ 2007, 76, 161310.

43. Moreels, I.; Lambert, K.; De Muynck, D.; Vanhaecke, F.; Poelman, D; Martins, J. C.; Allan, G.; Hens, Z. Composition and Size-Dependent Extinction Coefficient of Colloida PbSe Quantum Dots. Chem. Mater. 2007, 19, 6101-6106.

44. Soref, $R_{i ;}$ Bennett, B. Electrooptical Effects in Silicon. IEEE J. Quantum Electron. 1987, 23, 123-129.

45. Schroder, D.; Thomas, R.; Swartz, J. Free Carrier Absorption in Silicon. IEEE J. Solid-State Circuits 1978, 13, 180-187.

46. Kekatpure, R. D.; Brongersma, M. L. Quantification of FreeCarrier Absorption in Silicon Nanocrystals with an Optical Microcavity. Nano Lett. 2008, 8, 3787-3793.

47. Kekatpure, R. D.; Brongersma, M. L. Near-Infrared FreeCarrier Absorption in Silicon Nanocrystals. Opt. Lett. 2009 34, 3397-3399.

48. Robel, I.; Gresback, R.; Kortshagen, U.i Schaller, R. D.; Klimov, V. I. Universal Size-Dependent Trend in Auger Recombination in Direct-Gap and Indirect-Gap Semiconductor Nanocrystals. Phys. Rev. Lett. 2009, 102, 177404.

49. Klimov, V. I.; Ivanov, S. A.; Nanda, J.; Achermann, M.; Bezel, l.; McGuire, J. A.; Piryatinski, A. Single-Exciton Optical Gain in Semiconductor Nanocrystals. Nature 2007, 447, 441-446.

50. Talapin, D.; Murray, C. PbSe Nanocrystal Solids for N- and P-Channel Thin Film Field-Effect Transistors. Science 2005 310,86

51. Schaller, R. D.; Klimov, V. I. High Efficiency Carrier Multiplication in PbSe Nanocrystals: Implications for Solar Energy Conversion. Phys. Rev. Lett. 2004, 92, 186601.

52. Trinh, M. T.; Houtepen, A. J.; Schins, J. M.; Hanrath, T.; Piris, J.; Knulst, W.; Goossens, A. P. L. M.; Siebbeles, L. D. A. In Spite of Recent Doubts Carrier Multiplication Does Occur in PbSe Nanocrystals. Nano Lett. 2008, 8, 1713-1718.

53. Murray, C.; Sun, S.; Gaschler, W.; Doyle, H.; Betley, T.; Kagan C. Colloidal Synthesis of Nanocrystals and Nanocrystal Superlattices. IBM J. Res. Dev, 2001, 45, 47-56.

54. Moreels, l: Fritzinger, B: Martins, J. C.: Hens, Z. Surface Chemistry of Colloidal PbSe Nanocrystals. J. Am. Chem. Soc. 2008, 130, 15081-15086.

55. Pietryga, J.; Werder, D.; Williams, D.; Casson, J.; Schaller, R Klimov, V,; Hollingworth, J. Utilizing the Lability of Lead Selenide To Produce Heterostructured Nanocrystals with Bright, Stable Infrared Emission. J. Am. Chem. Soc. 2008 $130,4879-4885$. 